'C' RNA before treatment, during treatment and after treatment. For prospective study, 290 patients with Hepatitis C genotype 3 were studied for two years (June 2015 to June 2017). They were enrolled both from IPD and OPD. These patients were detected HCV positive during blood donation, preoperative evaluation, evaluation of liver function tests etc.

Results For the retrospective group, SVR24 was achieved in 90.96\% (171/188) patients on Peg-IFN alpha 2a plus Ribavirin (weight based) combination for a period of 24 weeks. For prospective group, SVR12 was achieved in 94.57\% (157/166) patients on Sofosbuvir plus Ribavirin combination for a period of 12 weeks and 98\% (98/100) patients on Sofosbuvir plus Daclatasvir combination for a period of 12 weeks among noncirrhotics while as among cirrhotics SVR24 was achieved in $83.33 \%(20 / 24)$ patients on Sofosbuvir plus Ribvirin plus Daclatasvir combination for a period of 24 weeks.

Conclusions Overall in the retrospective group, an SVR24 was $90.96 \%$, and in prospective group, SVR12 was achieved in $94.57 \%$ on Sofosbuvir plus Ribavirin combination for 12 weeks, $98 \%$ on Sofosbuvir plus Daclatasvir for 12 weeks and in Cirrhotic group, 83.33\% achieved an SVR12 on Sofosbuvir plus Ribvirin plus Daclatasvir combination for 24 weeks.

\section{IDDF2018-ABS-0186 CHOLEDOCHODUODENAL FISTULA IN A PATIENT WITH BILIARY INTRADUCTAL PAPILLARY MUCINOUS NEOPLASM}

${ }^{1}$ Lei Liu*, 'Like Bie, ${ }^{1} B$ in Xu, 'Wei Wang, ${ }^{3}$ Biao Gong. 'Department of Gastroenterology, Ruijin Hospital, Shanghai Jiaotong University School of Medicine, Shanghai, China; 2Department of Surgery, Ruijin Hospital, Shanghai Jiaotong University School of Medicine, Shanghai, China; ${ }^{3}$ Department of Gastroenterology, Shuguang Hospital of Shanghai University of Traditional Chinese Medicine, Shanghai, China

\subsection{6/gutjnl-2018-IDDFabstracts.228}

Background Biliary intraductal papillary mucinous neoplasm (IPMN-B) is an uncommon low malignant tumour of the bile duct. IPMN-B characterised by secreting mucus, biliary obstruction, dilation of the bile duct and has potential to transform to the malignancy. In this study, we may introduce our experiences and summarise the clinicopathological and molecular features of IPMN-B by literature review.

Methods Here we report the case of a 66-year-old female presented with repeated fever and elevated liver enzymes. Magnetic resonance cholangiopancreatography (MRCP) demonstrated definite cyst tumour and biliary dilatation of common bile duct and hepatic duct (figure 1). Endoscopic retrograde cholangiography (ERC) revealed that the bile duct filled with floating filling-defects and the mucus perforated to duodenal mucosa generating the choledoch duodenal fistula. Due to the poor clinical condition of this patient, we performed biliary plastic stents through the duodenal fistula and transcended the primary mucinous neoplasm to drainage the bile acid. We also summarise the clinicopathological and molecular features of IPMN-B by literature review, which may help to recognise this rare bile duct tumour and improve the clinical diagnosis and treatment decision when encountering the similar series cases.

Results We successfully relieved biliary obstruction and remitted the elevated liver enzymes and infectious symptoms of this patient by endoscopic therapy. In addition, the patient undergoing the follow-up to monitor the biliary mucinous neoplasm.

Conclusions Our case demonstrated that patients with IPMN-B or accompany with complications such as the choledochoduodenal fistula, palliative endoscopic drainage may be an effective approach. ERCP is useful for the diagnosis, drainage and decompression of biliary IPMN.

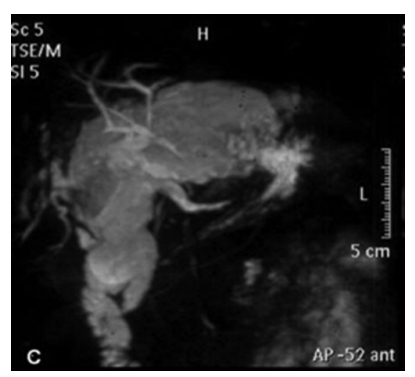

Abstract IDDF2018-ABS-0186 Figure 1 Median vitamin d levels according to child pugh status

\section{IDDF2018-ABS-0189 EFFECT OF DIABETES MELLITUS AND VITAMIN D LEVELS ON HEPATITIS C RELATED LIVER DISEASE}

${ }^{1}$ Varun Gupta*, ${ }^{2}$ Ashish Kumar, ${ }^{2}$ Praveen Sharma, ${ }^{2}$ Anil Arora. ${ }^{1}$ Fortis Memorial Research Institute Gurgaon, India; ${ }^{2}$ Sir Ganga Ram Hospital, New Delhi, India

\subsection{6/gutjnl-2018-IDDFabstracts.229}

Background There is a high incidence of Diabetes mellitus and vitamin D deficiency seen among Hepatitis C patients. The effect of the simultaneous occurrence of DM and low levels of 25-Hydroxy Vitamin D on HCV related liver disease remains unclear. In this study, we have aimed to assess the influence of DM and levels of Vitamin D on HCV related liver disease patients.

Methods In this retrospective study, all patients who tested positive for Anti-HCV between January 2014 - December 2016 were included and were reviewed for gender, age, diabetes, genotype, level of 25-Hydroxy Vitamin D, the status of liver disease including HCC at inclusion. The association of diabetes and low Vitamin D levels $(<30 \mathrm{ng} / \mathrm{mL})$ with liver status and child pugh class (CTP) was analysed.

Results A total of 777 patients were included. At presentation 439 (56\%) patients had cirrhosis; including 51 (7\%) with HCC and 287 (37\%) had chronic hepatitis. 25-Hydroxy Vitamin D level was available in 206 patients at baseline. Low Vitamin D level was present in [126/206 (61\%)] of the patients. DM was present in $[165 / 777(21 \%)]$ of the patients and was more common with genotype 1 and $4(p=0.056)$. Patients who had associated diabetes presented more commonly with cirrhosis 122/439 (28\%) and HCC 122/439 $(29 \%)$ in comparison to chronic hepatitis 28/287 (10\%) $(\mathrm{p}<0.01)$. Lower Vitamin D level $(<30 \mathrm{ng} / \mathrm{mL})$ was seen in $67 \%(98 / 147)$ of patients with cirrhosis, 78\% (11/14) with HCC and 47\% $(28 / 59)$ with chronic hepatitis $(p=0.012)$. In cirrhotic patients, vitamin $\mathrm{D}$ deficiency was significant in child 
B (72\%) and Child C (75\%) patients in comparison to child a patients $(52 \%)(\mathrm{p}=0.013)$ (figure 1$)$.

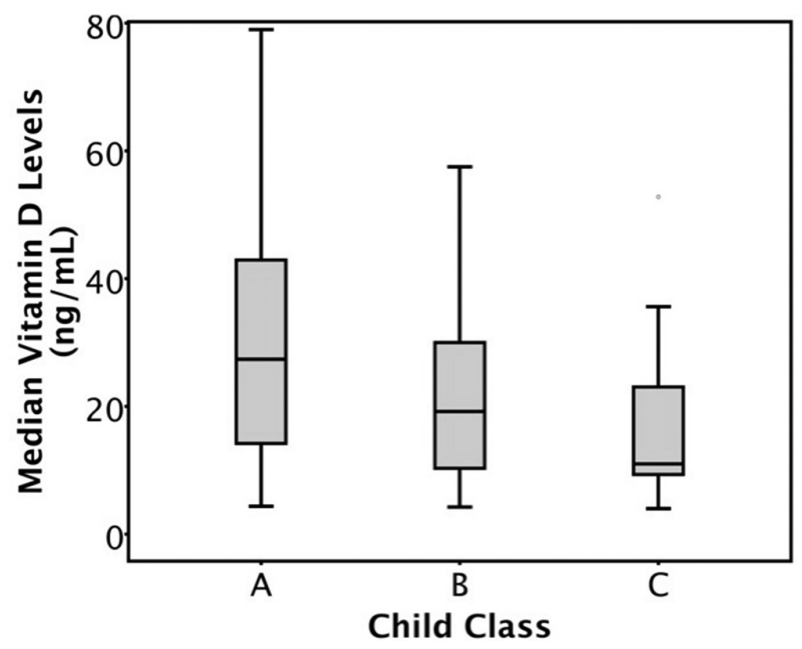

Abstract IDDF2018-ABS-0189 Figure 1 Median vitamin d levels according to child pugh status
Conclusions 25-Hydroxy Vitamin D deficiency was present in $61 \%$ and diabetes mellitus co-existed in $21 \%$ of HCV patients at presentation. Diabetes and low levels of $25-\mathrm{OH}$ vitamin $\mathrm{D}$ at presentation were associated with higher incidence of cirrhosis and HCC. We believe that improving diabetes control and correcting vitamin D deficiency can improve outcomes in hepatitis $\mathrm{C}$ related liver disease.

\section{IDDF2018-ABS-0190 PREDICTORS OF SHORT-TERM MORTALITY IN SEVERE ALCOHOLIC HEPATITIS AND USE OF STEROIDS IN CLINICAL PRACTICE: AN EXPERIENCE OUTSIDE CLINICAL TRIAL FROM INDIA}

Ravi Daswani*, Ashish Kumar, Shivam Khare, A Shrihari, Praveen Sharma, Naresh Bansal, Vikas Singla, Anil Arora. Sir Gangaram Hospital, New Delhi, India

\subsection{6/gutjnl-2018-IDDFabstracts.230}

Background Severe $\mathrm{AH}$ (SAH) is the most florid presentation of alcohol-related liver disease with high short-term mortality. Steroids have remained the mainstay of therapy despite the controversy. The aim of the present study was to assess the risk factors and compare the severity scores predicting 90 day

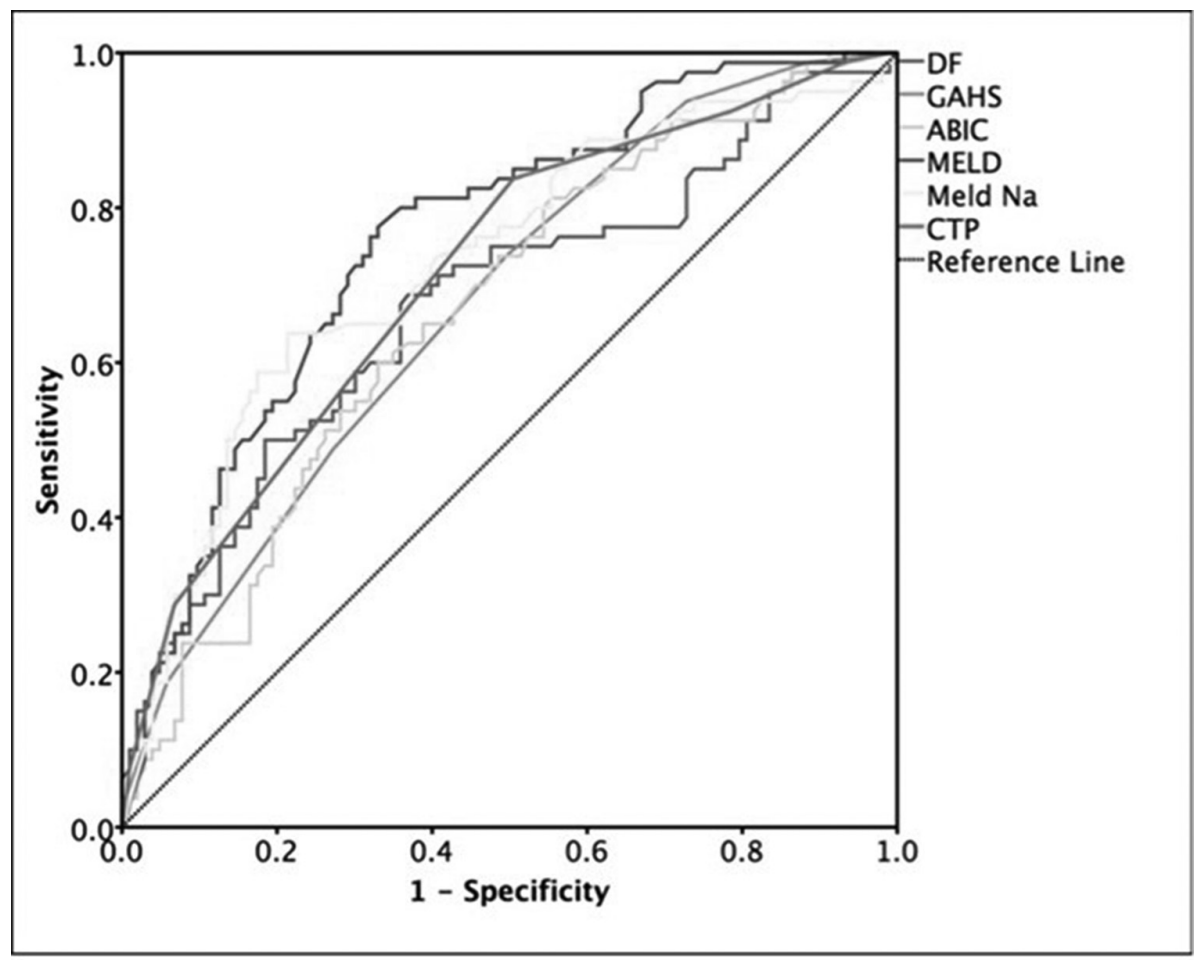

\begin{tabular}{|l|c|c|c|c|c|}
\hline \multirow{2}{*}{ Score } & \multirow{2}{*}{ AUC } & Std error & \multirow{2}{*}{ P value } & \multicolumn{2}{|c|}{$95 \% \mathrm{Cl}$} \\
\cline { 5 - 6 } & & & & Lower & Upper \\
\hline DF & 0.675 & 0.041 & $<0.001$ & 0.595 & 0.755 \\
\hline GAHS & 0.675 & 0.039 & $<0.001$ & 0.599 & 0.752 \\
\hline ABIC & 0.666 & 0.040 & $<0.001$ & 0.588 & 0.744 \\
\hline MELD & 0.761 & 0.035 & $<0.001$ & 0.691 & 0.830 \\
\hline Meld Na & 0.728 & 0.038 & $<0.001$ & 0.653 & 0.803 \\
\hline CTP & 0.713 & 0.038 & $<0.001$ & 0.638 & 0.788 \\
\hline
\end{tabular}

